\title{
Bovine apolipoprotein (apo)A-I displays more enhanced antioxidant and anti-atherosclerotic activity in lipid-free and lipid-bound states than human and porcine apoA-I
}

\author{
SEONG-MIN KIM ${ }^{1,2^{*}}$, GA-YOUNG PARK ${ }^{1,2^{*}}$, INHO CHOI $^{1,2}$ and $\mathrm{KYUNG}^{-H Y U N ~ C H O}{ }^{1,2}$ \\ ${ }^{1}$ School of Biotechnology and ${ }^{2}$ Research Institute of Protein Sensor, \\ Yeungnam University, Gyeongsan, Gyeongbuk 712-749, Republic of Korea
}

Received March 29, 2013; Accepted June 26, 2013

DOI: $10.3892 /$ ijmm.2013.1473

\begin{abstract}
Apolipoprotein A-I (apoA-I) is a major component of high-density lipoprotein (HDL), which displays anti-atherosclerotic activity in plasma. In the current study, we compared the functional and structural characteristics of human, bovine and porcine apoA-I as regards their antioxidant ability and protein stability. In the lipid-free state, the immunoreactivity of bovine and porcine apoA-I differed from that of human apoA-I and bovine and porcine apoA-I exhibited greater resistance to denaturation induced by urea treatment. Bovine apoA-I showed the weakest binding ability of dimyristoyl phosphatidylcholine; however, bovine apoA-I formed slightly larger reconstituted HDL (rHDL) particles with palmitoyl oleoyl phosphatidylcholine, with a higher number of apoA-Icontaining particles. Bovine and porcine apoA-I comprised of pentameric structures, whereas human apoA-I in the rHDL state consisted of trimeric structures. Although apoA-I from all three species showed a similar content of $\alpha$-helicity in the lipidfree state (approximately 53\%), bovine apoA-I showed a lower $\alpha$-helicity content (approximately 66\%) compared with human apoA-I (approximately 74\%) in the rHDL state. Bovine apoA-I was more resistant to denaturation and glycation upon treatment with urea and fructose, respectively. Furthermore, bovine apoA-I showed a greater inhibition of cupric ion-mediated lowdensity lipoprotein (LDL) oxidation and uptake of acetylated LDL by macrophages compared with human or porcine apoA-I in the lipid-free and lipid-bound states. In conclusion, bovine apoA-I has unique functional properties in the lipid-free and lipid-bound states, and displays significantly enhanced antiatherosclerotic activity.
\end{abstract}

Correspondence to: Professor Kyung-Hyun Cho, School of Biotechnology, Yeungnam University, 214-1 Dae-dong, Gyeongsan, Gyeongbuk 712-749, Republic of Korea

E-mail: chok@yu.ac.kr

${ }^{*}$ Contributed equally

Key words: apolipoprotein A-I, high-density lipoprotein, antioxidant, atherosclerosis

\section{Introduction}

Human apolipoprotein A-I (apoA-I), the major component of high-density lipoprotein (HDL), is a protein of 243 amino acids with repeated 22-residue homologous segments separated by prolines (1). HDL plays a critical role in reverse cholesterol transport (RCT), which is involved in the removal of excess cholesterol from peripheral cells and its delivery to the liver and steroidogenic cells for catabolism (2). In vertebrates, normal HDL exerts potent antioxidant and anti-inflammatory effects (3) and has anti-infective properties in plasma (4). It is well established that there are strong correlations between apoA-I structure and function, and the beneficial effects of HDL are highly dependent on its apoA-I content and conformation. Lipid-free apoA-I, as well as apoA-I in reconstituted HDL (rHDL) particles, may stimulate insulin secretion from pancreatic $\beta$-cells (5). These data raise the possibility that lipid-free apoA-I may play a unique role in the mammalian health system.

As a tool for gene delivery, we previously reported that the efficiency of viral delivery and adenovirus stability can be significantly enhanced by applying proteoliposomes (PLs) containing apoA-I to human cells and zebrafish models (6). Recently, a PL containing apoA-I and its mutant (V156K) was shown to enhance the rapid tumor regression activity of human origin oncolytic adenovirus in tumor-bearing zebrafish and nude mice (7). These studies raise the possibility that apoA-I may be applied to the generation of vehicles for the delivery of pharmaceuticals, genes and vaccines.

Over the past three decades, the characterization of apoA-I and its mutants from a variety of sources in both the lipid-free and lipid-bound states has been extensively attempted with purified apoA-I from human plasma, animal plasma, and/or various recombinant apoA-I produced in an E. coli-based expression system $(1,8)$. As a result, many functional-structural domains have been elucidated, as described in the study by Bashtovyy et al (9). Phylogenetic comparisons of apoA-I sequences have been described in the study by Brouillette et al (10), as they observed that the deletion of a single residue in exon 4 of the pig and cow did not drastically alter the straight alignment of the hydrophobic face, whereas the rat and mouse displayed several amino acid deletions in exon 4 that altered the straight hydrophobic face. 
However, there has been no direct comparison of the functional and structural properties of human apoA-I with those of major vertebrates in domestic animals, such as pigs or cattle. In the present study, we compared the functional characteristics of human, bovine and porcine apoA-I in plasma in the lipid-free and lipid-bound states, as regards their antioxidant and anti-atherosclerotic activities.

\section{Materials and methods}

Materials. Dimyristoyl phosphatidylcholine (DMPC, \#850345) and palmitoyl oleoyl phosphatidylcholine (POPC, \#850457) were obtained from Avanti Polar Lipids, Inc. (Alabaster, AL, USA). Bis(sulfosuccinimidyl) suberate (BS ${ }_{3}$, \#S5799) and sodium cholate (\#C1254) were purchased from Sigma (St. Louis, MO, USA). $\alpha$-linolenic acid (all-cis-9,12,15-octadecatrienoic acid, 18:3, \#L2376) was purchased from Sigma. Bovine and porcine plasma was obtained from the Bovine Genome Resources Bank of Korea at Yeungnam University (Gyeongsan, Korea) and a local slaughter house (Gyeongsan, Korea), respectively, with the addition of EDTA (final concentration, $1 \mathrm{mM}$ ) as an anti-coagulant.

Purification of HDL and apoA-I. HDL and apoA-I were purified from human, bovine and porcine plasma using ultracentrifugation and column chromatography, according to a previously described method (11). The $\mathrm{NH}_{2}$-terminal aminoacid sequence of apoA-I was determined using an Applied Biosystems Procise 491 HT protein sequencer (Applied Biosystems, Foster City, CA, USA) located in the Seoul Branch of the Korea Basic Research Institute (Seoul, Korea).

DMPC clearance assay. The interactions of apoA-I from each species with DMPC $(3.5 \mathrm{mg} / \mathrm{ml})$ were monitored by the method described in the study by Pownall et al (12) with slight modifications. The mass ratio of DMPC to protein was 2:1 $(w / w)$ in a total reaction volume of $0.76 \mathrm{ml}$.

Purification and oxidation of low-density lipoprotein (LDL). LDL (density, $>1.019$ to $<1.063$ ) was purified from healthy human plasma (Blood Bank of Yeungnam University Medical Center, Daegu, Korea) by ultracentrifugation $(100,000 \mathrm{x} \mathrm{g})$ for $22 \mathrm{~h}$ at $4^{\circ} \mathrm{C}$. Oxidized LDL (oxLDL) was produced by incubating the LDL fraction with copper sulphate $\left(\mathrm{CuSO}_{4}\right)$ (final concentration, $10 \mu \mathrm{M}$ ) for $4 \mathrm{~h}$ at $37^{\circ} \mathrm{C}$. The oxLDL was then filtered $(0.2 \mu \mathrm{m}$ filter $)$ and analyzed by thiobarbituric acid reactive substances (TBARS) assay to determine the extent of oxidation, as previously described (13).

Synthesis of reconstituted HDL. Discoidal rHDL was prepared using the sodium cholate dialysis method $(14,15)$ with an initial molar ratio of POPC:cholesterol:apoA-I:sodium cholate of 95:5:1:150. The size and hydrodynamic diameter of rHDL particles were determined by $8-25 \%$ native polyacrylamide gradient gel electrophoresis (PAGGE; Pharmacia PhastSystem) by comparison with standard globular proteins (GE Healthcare, Uppsala, Sweden).

$B S_{3}$-cross-linking. The number of apoA-I molecules per rHDL particle, as well as the self-association properties of lipid- free proteins, were determined by cross-linking with $\mathrm{BS}_{3}$ as previously described (16), followed by the analysis of reaction products by sodium dodecyl sulfate-polyacrylamide gel electrophoresis (SDS-PAGE) on pre-cast 8-25\% gradient gels (GE Healthcare).

Circular dichroism $(C D)$. The average $\alpha$-helix contents of proteins in the lipid-free and lipid-bound states were measured by CD spectroscopy using a J-715 Spectropolarimeter (Jasco, Tokyo, Japan). Spectra were obtained from 250-190 nm at $25^{\circ} \mathrm{C}$ in a $0.1-\mathrm{cm}$ path-length quartz cuvette at a bandwidth of $1.0 \mathrm{~nm}$, a speed of $50 \mathrm{~nm} / \mathrm{min}$, and a $4-\mathrm{sec}$ response time. The protein samples were diluted to $0.07 \mathrm{mg} / \mathrm{ml}$ so that lipid-free proteins could avoid apolipoprotein self-association, whereas lipid-bound proteins were diluted to $0.1 \mathrm{mg} / \mathrm{ml}$. Four scans were accumulated and averaged. The $\alpha$-helix content was calculated from the molar ellipticity at $222 \mathrm{~nm}$ as previously described (17).

Characterization of tryptophan (Trp) fluorescence and isothermal denaturation. The wavelength of maximum fluorescence (WMF) of Trp residues in apoA-I protein was determined from uncorrected spectra obtained using an LS55 spectrofluorometer (Perkin-Elmer, Norwalk, CT, USA) in conjunction with the WinLab software package 4.00 (Perkin-Elmer) and a 1-cm path-length Suprasil quartz cuvette (Fisher Scientific, Pittsburgh, PA, USA). Briefly, the samples were excited at $295 \mathrm{~nm}$ to avoid tyrosine fluorescence, and the emission spectra were then scanned from $305-400 \mathrm{~nm}$ at room temperature. The effects of the addition of urea on the secondary structure of apoA-I in the lipid-bound state were monitored by observing the WMF as described in our previous study (15).

Glycation of apoA-I in lipid-free and lipid-bound state. In order to compare protein glycation, apoA-I from each species in the lipid-free or lipid-bound state was incubated with D-fructose (final concentration, $250 \mathrm{mM}$ ) as described our previous study (18). apoA-I was incubated for upto $72 \mathrm{~h}$ under air gas containing $5 \% \mathrm{CO}_{2}$ at $37^{\circ} \mathrm{C}$. The extent of advanced glycation reactions was determined from reading the fluorometric intensity at $370 \mathrm{~nm}$ (excitation) and $440 \mathrm{~nm}$ (emission), as described previously (18).

Western blot analysis. To compare the cross-reactivity of each apoA-I protein with antibodies specific to the human variant, equal amounts $(2.5 \mu \mathrm{g}$ of protein) of apoA-I were loaded and electrophoresed on 15\% SDS-PAGE gels and detected by anti-human full-length apoA-I goat antibody (ab7613; Abcam, Cambridge, UK) and donkey anti-goat immunoglobulin G-horseradish peroxidase (HRP) (SC2020; Santa Cruz Biotechnology, Inc., Santa Cruz, CA, USA) as the secondary antibody (diluted 1:2,000). The apoA-I protein concentration in the rHDL and lipid-free states was determined using the Lowry assay as modified by Markwell et al (19) with bovine serum albumin as the standard.

Inhibition of $L D L$ oxidation. In order to determine the extent of oxidation, purified human LDL was incubated with $10 \mu \mathrm{M}$ $\mathrm{CuSO}_{4}$ for up to $3 \mathrm{~h}$ in the presence of apoA-I in the lipidfree or rHDL state (final $2 \mu \mathrm{M}$ of protein). During incubation, 

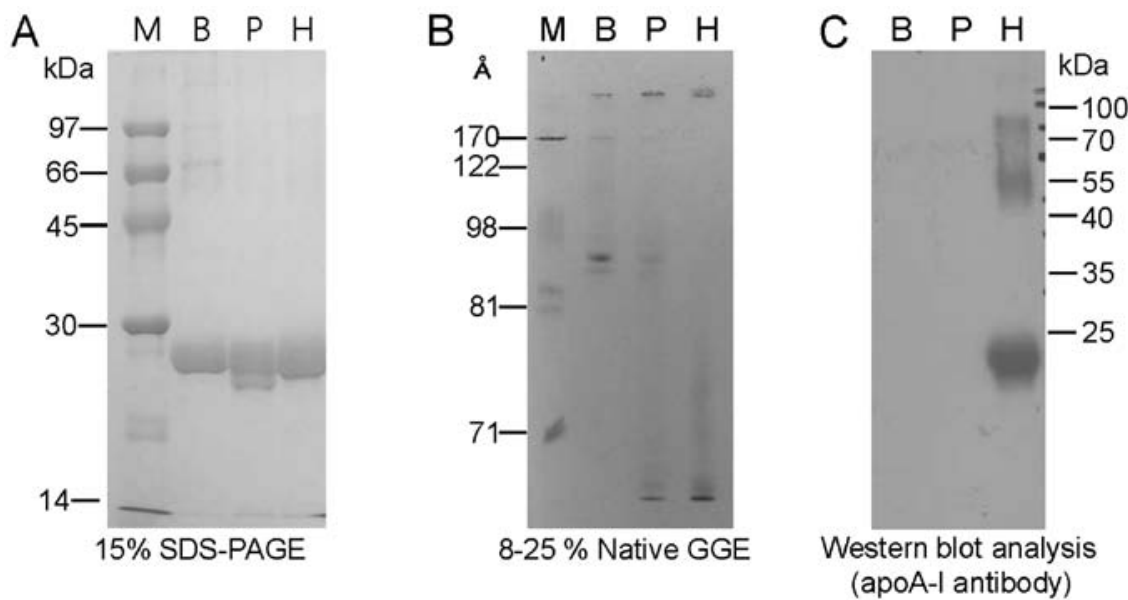

Figure 1. Electrophoretic mobility of three mammalian apolipoprotein A-I (apoA-I) variants in the lipid-free state. Lane B, bovine apoA-I; lane P, porcine apoA-I; lane H, human apoA-I. (A) The purity of each apoA-I variant was assessed by $15 \%$ gel electrophoresis. M, molecular weight (standard). (B) Electromobility of apoA-I in 8-25\% native gradient gel electrophoresis (GGE). M, molecular weight (standard) (GE Healthcare, high-range standard). (C) Immunodetection of apoA-I using anti-human full-length apoA-I antibody (Abcam, Ab7613).

the quantity of conjugated dienes formed was monitored by following the absorbance at $234 \mathrm{~nm}\left(\mathrm{~A}_{234}\right)$ at $37^{\circ} \mathrm{C}(20)$ using a Beckman DU 800 spectrophotometer (Beckman Coulter, Inc., Fullerton, CA, USA) equipped with a MultiTemp III Thermocirculator (Amersham Biosciences, Uppsala, Sweden).

To verify the spectroscopic data, oxidized samples were subjected to electrophoresis on $0.5 \%$ agarose gels in order to compare their electromobilities as previously described (21); migration of each lipoprotein is known to depend on its intact charge and size. The gels were then dried and the bands were stained with $0.125 \%$ Coomassie Brilliant Blue.

Acetylation of LDL. The acetylation of LDL (acLDL) was performed using saturated sodium acetate and acetic anhydride according to a previously described method (22). Following acetylation and subsequent dialysis, the acLDL protein content was determined and filtered through a $0.22 \mu \mathrm{m}$ filter (Millex; Millipore, Bedford, MA, USA). To visualize the phagocytosis of acLDL, a fluorescent cholesterol derivative [22-(N-7-nitrobenz-2-oxa-1,3-diazol-4-yl)amino-23,24-bisnor5-cholen-3-ol (NBD-cholesterol), Molecular Probes N-1148; $70 \mu \mathrm{g}$ of NBD-cholesterol/mg of apoA-I] was added to the acLDL particles.

LDL phagocytosis assay. THP-1 cells, a human monocytic cell line, were obtained from the American Type Culture Collection (ATCC; \#TIB-202 ${ }^{\mathrm{TM}}$; Manassas, VA, USA) and maintained in RPMI-1640 medium (HyClone, Logan, UT, USA) supplemented with $10 \%$ fetal bovine serum (FBS) until needed for experimentation. Cells that had undergone no more than 20 passages were incubated in medium containing phorbol 12-myristate 13-acetate (PMA; final concentration, $150 \mathrm{nM}$ ) in 24-well plates for $24 \mathrm{~h}$ at $37^{\circ} \mathrm{C}$ in a humidified incubator (5\% $\mathrm{CO}_{2}, 95 \%$ air) to induce differentiation into macrophages. The differentiated and adherent macrophages were then rinsed with warm PBS and incubated with $400 \mu \mathrm{l}$ of fresh RPMI-1640 medium containing 1\% FBS, $50 \mu \mathrm{l}$ of (acLDL; $1 \mathrm{mg}$ of protein $/ \mathrm{ml}$ in PBS), and $50 \mu 1$ of PBS or each protein (final $2 \mu \mathrm{M})$ for $48 \mathrm{~h}$ at $37^{\circ} \mathrm{C}$ in a humidified incubator. Following incubation, the cells were washed with PBS three times and then fixed in $4 \%$ paraformaldehyde for $10 \mathrm{~min}$. The fixed cells were then stained with oil Red O staining solution (0.67\%) and then washed with distilled water. THP-1 macrophage-derived foam cells were then observed and photographed using a Nikon Eclipse TE2000 microscope (Nikon, Tokyo, Japan) at x600 magnification.

Statistical analysis. All data are expressed as the means \pm SD of at least three independent experiments with duplicate samples. Comparisons between the results were made using the Student's t-test with SPSS software (version 12.0; SPSS, Inc., Chicago, IL, USA). A P-value $<0.05$ was considered to indicate a statistically significant difference.

\section{Results}

Identification of apoA-I and sequence comparisons. Although apoA-I from all species ( $>95 \%$ purity) exhibited $28-29 \mathrm{kDa}$ molecular weight in the lipid-free state from SDS-PAGE (Fig. 1A), bovine apoA-I showed the highest position with the slowest mobility in the native gel electrophoresis (Fig. 1B).

$\mathrm{N}$-terminal amino acid sequencing confirmed the identities of bovine (lane B) and porcine (lane P) apoA-I. In each protein, 16 amino acids were identical to the GenBank sequences for bovine (ID AAI02942.1) and porcine (ID CAA49234.1) apoA-I. Bovine and porcine apoA-I differed from the human protein in their 2nd amino acid (Asp), which corresponds to Glu; the 4th Pro in the human sequence was deleted from the bovine and porcine proteins. Furthermore, Pro7 in human apoA-I was replaced by Ser in bovine apoA-I; the 14th residue, leucine, in human apoA-I was replaced by Phe in the bovine and porcine variants (data not shown).

Immunodetection of bovine and porcine apoA-I. The human specific antibody for apoA-I could not detect bovine or porcine protein $(2.5 \mu \mathrm{g} /$ lane $)$, suggesting that the antigenic epitope was dissimilar between the species (Fig. 1C). The comparison of the amino acid sequences revealed the deletion of the 
Table I. Compositional and spectroscopic analyses of apoA-I from bovine, porcine and human plasma in the lipid-bound state $(\mathrm{rHDL})^{\mathrm{a}}$.

\begin{tabular}{lccc}
\hline & Bovine & Porcine & Human \\
\hline $\mathrm{WMF}^{\mathrm{b}}(\mathrm{nm})$ at urea 0 M & $341 \pm 3(348 \pm 1)^{\mathrm{c}}$ & $341 \pm 2(346 \pm 2)$ & $344 \pm 2(348 \pm 2)$ \\
$\mathrm{WMF}^{\mathrm{n}}(\mathrm{nm})$ at urea 7 M & $354 \pm 2(355 \pm 1)$ & $357 \pm 2(359 \pm 2)$ & $355 \pm 1(357 \pm 2)$ \\
$\alpha$-helicity $\left.^{\mathrm{d}} \%\right)$ & $66 \pm 3(53 \pm 4)$ & $70 \pm 4(55 \pm 3)$ & $74 \pm 2(53 \pm 3)$ \\
Size $^{\mathrm{e}}(\AA)$ & 105 & 101 & 98,80 \\
No. of apoA-I $^{\mathrm{f}} / \mathrm{rHDL}$ & $2-5$ & $2-5$ & 2,3 \\
$\mathrm{pI}^{\mathrm{g}}($ calculated & 5.32 & 5.11 & 5.24 \\
\hline
\end{tabular}

${ }^{a}$ rHDL was synthesized with palmitoyl oleoyl phosphatidylcholine (POPC):cholesterol:apoA-I:sodium cholate at a molecular ratio of 95:5:1:150, respectively. ${ }^{b}$ Determined from fluorospectroscopy (excitation, $295 \mathrm{~nm}$; emission, 305-400 nm). ${ }^{\mathrm{c}}$ Numbers in parentheses indicate proteins in the lipid-free state. ${ }^{\mathrm{d}}$ Determined from circular dichroic spectroscopy. ${ }^{\mathrm{e}}$ Determined from $8-25 \%$ native-gradient gel electrophoresis with densitometric scanning analysis. ${ }^{\mathrm{f}}$ Determined from $\mathrm{BS}_{3}$-cross-linking and 8-25\% SDS-PAGGE. 'Isoelectric point (pI) was calculated using protean module of DNASTAR software (version 5.07).
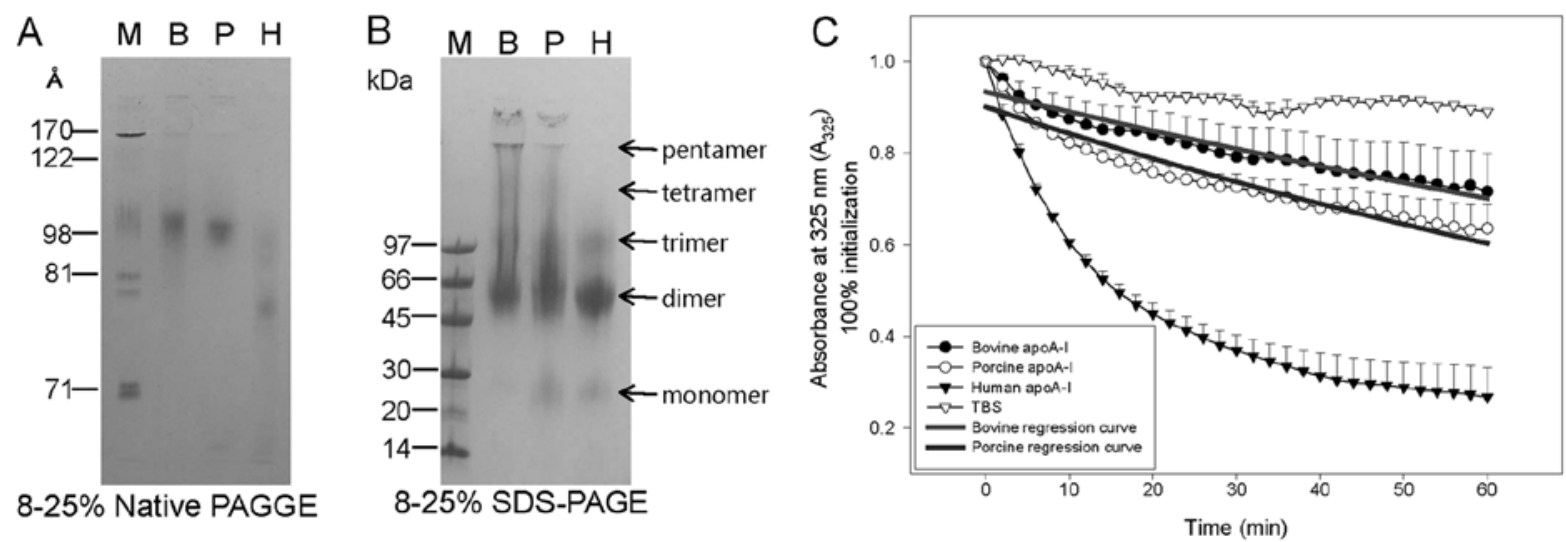

Figure 2. Electrophoretic mobility of three mammalian apolipoprotein A-I (apoA-I) variants in the lipid-bound state. Lane B, bovine apoA-I; lane P, porcine apoA-I; lane H, human apoA-I. (A) Electromobility of apoA-I without sample boiling. M, molecular weight (standard) (GE Healthcare, high-range standard). (B) Bis-sulfosuccinimidyl suberate $\left(\mathrm{BS}_{3}\right.$ )-cross-linked apoA-I in the POPC-rHDL state, as visualized by Coomassie blue staining. M, molecular weight (standard) (Bio-Rad, low-range standard). (C) Interaction of each apoA-I variant with dimyristyol phosphatidylcholine (DMPC) multilamellar liposomes. The mass ratio of DMPC to protein was 2:1 (wt/wt) in a total reaction volume of $0.76 \mathrm{ml}$. POPC, palmitoyl oleoyl phosphatidylcholine; rHDL, reconstituted high-density lipoprotein .

4th amino acid in the human protein, Pro, in the bovine and porcine apoA-I sequences; the loss of this residue may have disrupted the epitope. Pro4 and Ala187 in the human protein sequence were deleted in bovine apoA-I; Pro4, Glu120 and Asn184 were deleted in porcine apoA-I.

Characterization of rHDL. Electrophoretic analysis of POPC-rHDL-associated apoA-I demonstrated that the major particle size ranged from 98-105 A (Fig. 2A). The bovine apoA-I-containing particles were the largest, at approximately $105 \AA$ in diameter. $\mathrm{BS}_{3}$-cross-linking revealed that bovine and porcine apoA-I rHDL contained up to five apoA-I units per particle, whereas human apoA-I rHDL contained three apoA-I units/particle (Fig. 2B).

DMPC clearance. In order to compare the phospholipid and polyunsaturated fatty acid binding capacity of the apoA-I, we monitored the clearance speed of the lipid emulsion. Bovine and porcine apoA-I displayed a much weaker phospholipid- binding ability compared with human apoA-I; values for the repair half-time $\left(\mathrm{T}_{1 / 2}\right)$ values were calculated at around 81 and $66 \mathrm{~min}$, respectively, from the regression curve (Fig. 2C). The phospholipid-binding ability of human apoA-I was much more pronounced as the approximate $T_{1 / 2}$ value was around $17 \mathrm{~min}$.

Spectroscopic analyses of secondary structure. In the lipidfree state, human and bovine apoA-I showed a WMF of 347 and $348 \mathrm{~nm}$, respectively, whereas porcine apoA-I showed a WMF of $345 \mathrm{~nm}$. Bovine and porcine proteins contained Trp residues at the 7th, 49th, 71st and 107th positions. In the rHDL state, the WMF apoA-I from all species demonstrated a blue shift of 3-4 nm (Table I). Secondary structure determination revealed $\alpha$-helicity in human apoA-I of 53 and $74 \%$ in the lipidfree and rHDL states, respectively, which was a $21 \%$ increase. However, bovine apoA-I showed 53 and $66 \% \alpha$-helicity in the lipid-free and rHDL states, respectively, indicating the lowest increase in $\alpha$-helicity (approximately 13\%). Porcine apoA-I 
A

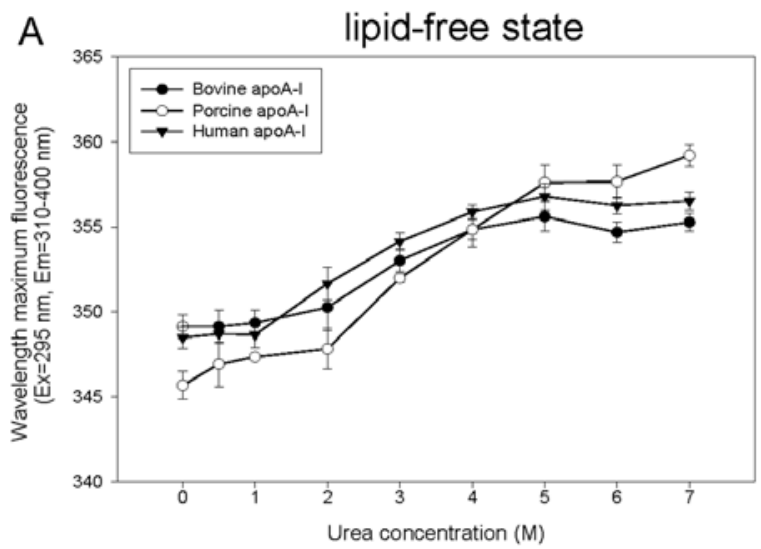

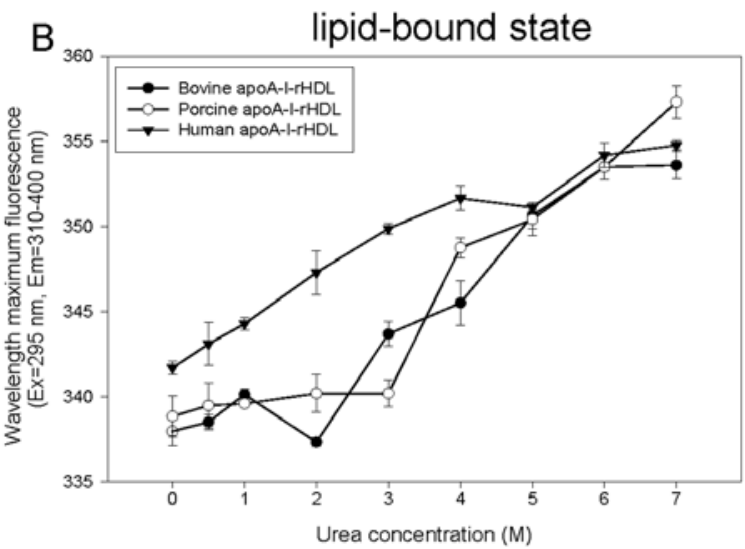

Figure 3. Denaturation behaviors of each apolipoprotein A-I (apoA-I) variant in (A) lipid-free and (B) lipid-bound states in the presence of urea. Monitoring of wavelength of maximum fluorescence (WMF) was performed using an LS55 Spectrofluorometer. Each sample was excited at 295 nm to avoid tyrosine fluorescence, and emission spectra were scanned from 305-400 $\mathrm{nm}$ at room temperature.
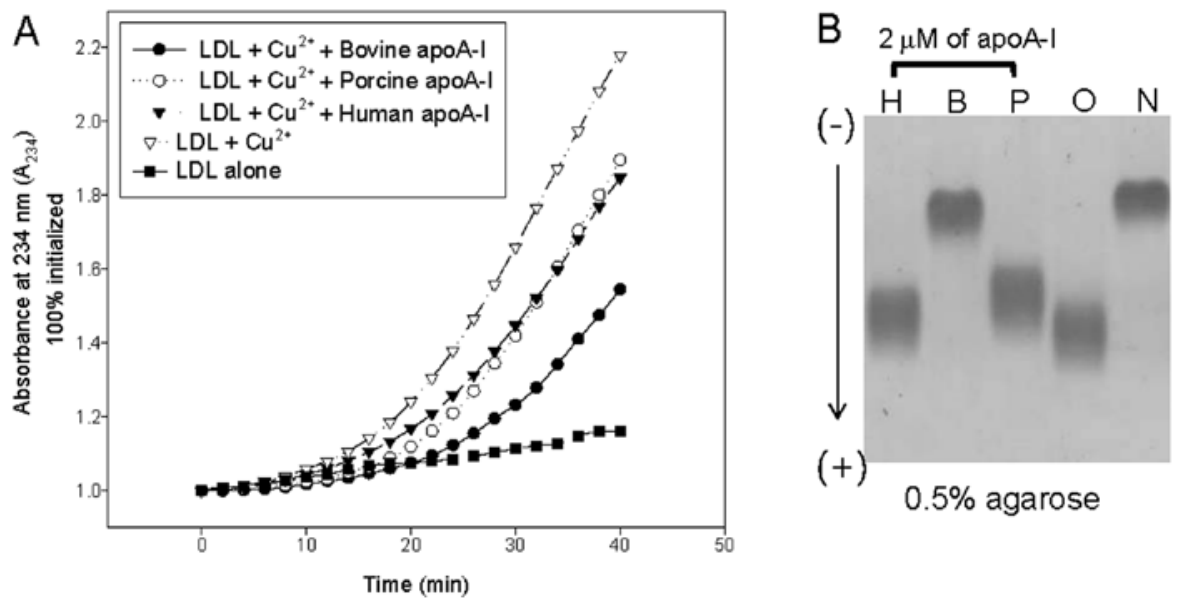

Figure 4. Apolipoprotein A-I (apoA-I) inhibition of cupric ion-mediated low-density lipoprotein (LDL) oxidation. (A) Conjugated diene detection during copper-mediated LDL oxidation. (B) Relative electrophoretic mobility profiles of LDL samples oxidized in the presence of $\mathrm{Cu}^{2+}$. Lane $\mathrm{N}$, native $\mathrm{LDL}(15 \mu \mathrm{g}$ of protein, without $\mathrm{Cu}^{2+}$ ); lane $\mathrm{O}$, oxidized $\mathrm{LDL}\left(\right.$ with $\left.\mathrm{Cu}^{2+}\right)$; lane $\mathrm{B}, \mathrm{LDL}+\mathrm{Cu}^{2+}+$ bovine apoA-I; lane $\mathrm{P}, \mathrm{LDL}+\mathrm{Cu}^{2+}+$ porcine apoA-I; lane $\mathrm{H}, \mathrm{LDL}+\mathrm{Cu}^{2+}$ + human apoA-I.

exhibited 55 and $70 \% \alpha$-helicity in the lipid-free and rHDL states, respectively, which was a $15 \%$ increase in $\alpha$-helicity.

Denaturation under urea treatment. In the lipid-free state, porcine and bovine apoA-I were resistant to denaturation at a urea concentration $<2 \mathrm{M}$ (Fig. 3A). Exposure to $7 \mathrm{M}$ urea stimulated the highest WMF of porcine apoA-I, whereas bovine and human apoA-I showed their highest WMF upon treatment with $5 \mathrm{M}$ urea. These results suggest that porcine and bovine apoA-I show variable sensitivity upon complete Trp exposure in the aqueous phase due to protein denaturation. In the rHDL state, porcine and bovine apoA-I were resistant to denaturation at a urea concentration $<5 \mathrm{M}$. Human apoA-I was the most susceptible to urea-induced denaturation (Fig. 3B).

Inhibition of LDL oxidation. In the lipid-free state, bovine apoA-I was the most resistant to cupric ion-mediated LDL oxidation during $40 \mathrm{~min}$ of incubation (Fig. 4A) (final concentration of each apoA-I, $2 \mu \mathrm{M}$ ); bovine apoA-I demonstrated an increase in $\mathrm{A}_{234}$ of $54 \%$, whereas porcine and human apoA-I were inhibited to a lesser degree (increase in $\mathrm{A}_{234}$ of $84-89 \%$ ).
Agarose electrophoresis revealed that LDL treated with bovine apoA-I (final $2 \mu \mathrm{M}$ ) migrated the slowest. This result suggests that LDL treated with bovine apoA-I was more resistant to the oxidation, as more oxLDL migrates faster due to an increase in the negative charge and smaller particle size (Fig. 4B).

Inhibition of acLDL uptake by macrophages. We monitored the uptake of acLDL into THP-1 cells in the presence of each apoA-I (Fig. 5) relative to the PBS-treated control (Fig. 5A) based on the detection of a fluorescent cholesterol derivative. In the lipid-free state, bovine apoA-I demonstrated the greatest inhibition of acLDL phagocytosis. Specifically, in the presence of lipid-free bovine apoA-I, the fluorescence decreased by $56 \%$ compared with the acLDL control (Fig. 5).

Glycation sensitivity of apoA-I. By fructosylation, apoA-I is usually prone to an increase in the WMF by the glycation process via the modification of its tertiary structure, resulting in the exposure of Trp in the aqueous phase. In the lipid-free state, porcine and human apoA-I showed an increase in WMF of approximately $2 \mathrm{~nm}$ during $72 \mathrm{~h}$ of incubation (Fig. 6A). 

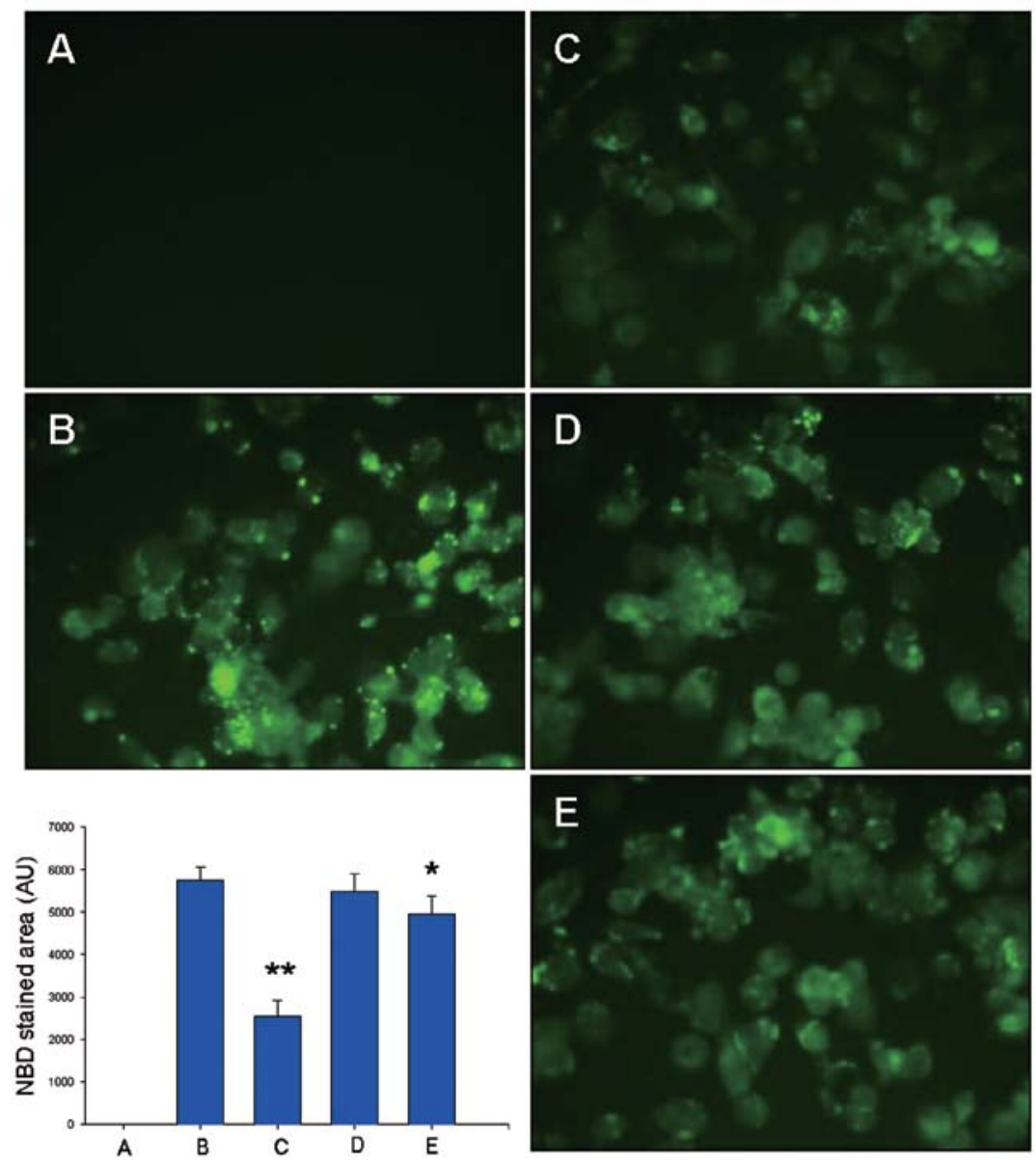

Figure 5. Inhibition of acetylated low-density lipoprotein (acLDL) phagocytosis by lipid-free apolipoprotein A-I (apoA-I) (final $2 \mu \mathrm{M}$ ). Differentiated THP-1 cells were incubated with each apoA-I (28 $\mu \mathrm{g}, 50 \mu \mathrm{l})$ for $48 \mathrm{~h}$ with or without $50 \mu \mathrm{l}$ of acLDL (50 $\mu \mathrm{g}$ protein). (A) PBS alone; (B) acLDL alone; (C) bovine apoA-I + acLDL; (D) porcine apoA-I + acLDL; (E) human apoA-I + acLDL. Inset graph shows quantification of fluorescence in cells using computerassisted morphometry for $0.8 \mathrm{~mm}^{2}$ windows. Data shown are the means $\pm \mathrm{SD}$ from three independent experiments performed in duplicate. ${ }^{* *} \mathrm{P}<0.001 \mathrm{vs}$. (B); ${ }^{*} \mathrm{P}<0.05$ vs. (B).
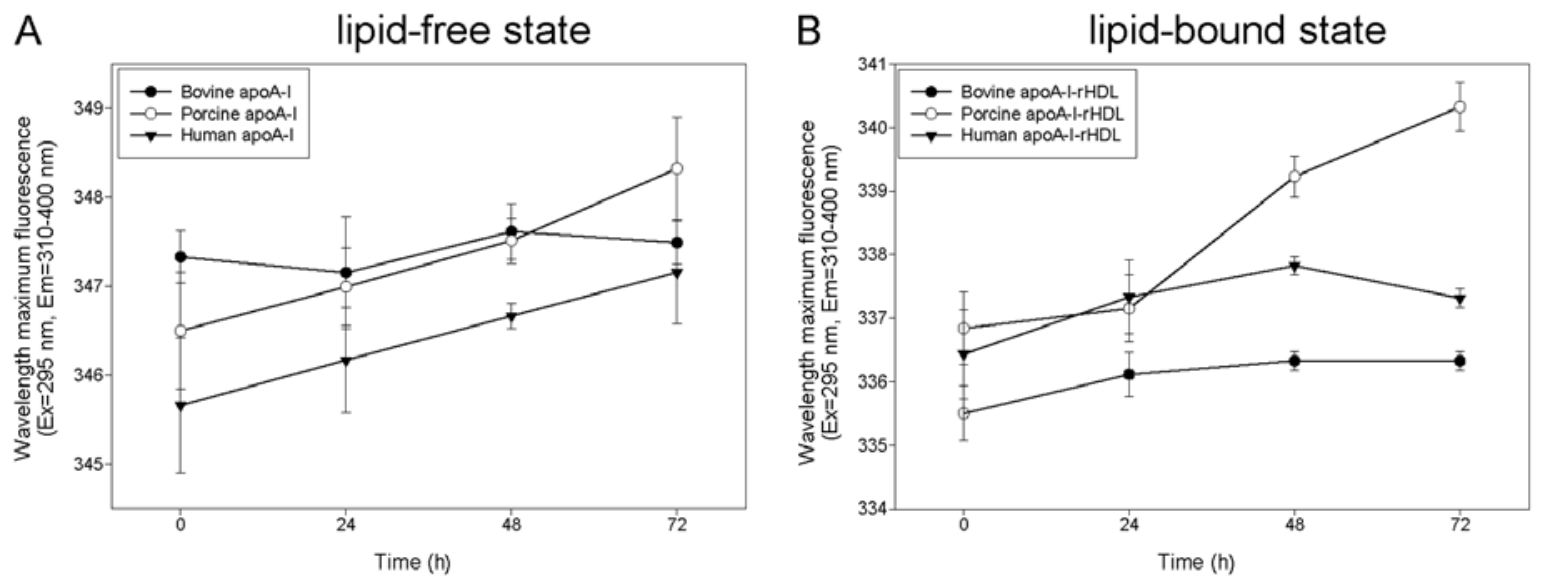

Figure 6. Comparison of sensitivity to glycation in (A) lipid-free and (B) lipid-bound states. Each apolipoprotein A-I (apoA-I) was treated with $1 \mathrm{mg} / \mathrm{ml}$ fructose (final concentration, $250 \mathrm{mM}$ ) at $37^{\circ} \mathrm{C}$ for $72 \mathrm{~h}$ in air containing $5 \% \mathrm{CO}_{2}$.

However, bovine apoA-I did not show any change in WMF in the lipid-free state (approximately $347 \mathrm{~nm}$ ). Although apoA-I has four $\operatorname{Trp}$ in the protein sequence, the sensitivity to fructosylation differed between the species. In the rHDL state, bovine apoA-I and human apoA-I showed the lowest increase in WMF (approximately $1 \mathrm{~nm}$ ) during $72 \mathrm{~h}$ of incubation (from 335 to $336 \mathrm{~nm}$ ), while porcine apoA-I showed the highest increase (approximately $3 \mathrm{~nm}$; from 337 to $340 \mathrm{~nm}$ ) (Fig. 6B). These results suggest that bovine apoA-I is the most resistant to glycation; however, the mechanisms invovled remain to be elucidated. 


\section{Discussion}

In the current study, we found several properties unique to bovine apoA-I compared with other mammalian apoA-I (human and porcine). Bovine apoA-I exhibited the weakest binding ability with DMPC (Fig. 2C) and the weakest sensitivity to denaturation by urea (Fig. 3). Bovine apoA-I was the most resistant to glycation with inhibition against LDL oxidation (Fig. 4) and foam cell formation via the phagocytosis of macrophages (Fig. 5).

apoA-I is a major component of HDL particles, which have strong antioxidant, anti-inflammatory and anti-infective properties (23). The overexpression of apoA-I in mice elicits resistance to diet-induced atherosclerosis (24). These studies raise the possibility of protein adaptation for the development of anti-atherosclerotic and anti-diabetic therapeutics. Serum HDL cholesterol and apoA-I levels are inversely associated with diabetic retinopathy, and serum apoA-I and apo-B are both strong biomarkers of this pathology. The infusion of rHDL with wild-type apoA-I or several mutant variants of apoA-I has been reported as an emerging therapy for acute regression $(25,26)$. These results suggest that apoA-I has potent therapeutic activities for the treatment of chronic metabolic diseases, such as diabetes and atherosclerosis.

The apoA-I amino acid sequence is conserved in higher vertebrates, such as pigs and cattle. Bovine apoA-I (GenBank ID AAI02942.1) and porcine apoA-I (GenBank ID CAA49234.1) consist of 241 and 240 amino acids, respectively, in their mature sequences. Furthermore, they exhibit $78 \%$ (bovine) or $80 \%$ (porcine) sequence identity to the human protein (AAH05380.1). Due to these amino acid differences, it is possible that the location of antigenic amino acids varies according to species. Collet et al (27) reported that several monoclonal antibodies (mAbs) against human apoA-I are unable to detect porcine or bovine protein, suggesting that apoA-I immunoreactivity may differ among species. They also tested 20 different $\mathrm{mAbs}$ against apoA-I proteins from humans, pigs and cattle. Between humans and pigs, only five mAbs displayed 50-100\% immunoreactivity. On the other hand, between cattle and humans, only one mAb displayed 50-100\% immunoreactivity with two coincidental epitope regions around amino acids 23-29 and amino acids 60-82.

Secondary structure determination revealed $\alpha$-helicity in human apoA-I of 53 and $74 \%$ in the lipid-free and rHDL states, respectively, which was a $21 \%$ increase. However, bovine apoA-I showed 53 and $66 \% \alpha$-helicity in the lipid-free and rHDL states, respectively, indicating a $13 \%$ increase in $\alpha$-helicity. Porcine apoA-I exhibited 55 and $70 \% \alpha$-helicity in the lipid-free and rHDL states, respectively, which was a $15 \%$ increase in $\alpha$-helicity. These results indicate that the $\alpha$-helicity of individual apoA-I from bovine or porcine plasma is lower than that isolated from human plasma. The inclusion of more bovine or porcine apoA-I molecules into rHDL (Fig. 2B) suggests that additional $\alpha$-helicity is non-essential. Another interpretation of this finding is that more bovine or porcine apoA-I molecules are required for rHDL formation due to their lower $\alpha$-helicity.

As regards the structural and functional analysis of apoA-I, Jonas and Krajnovich (28) reported that bovine apoA-I displays a blue shift of approximately $10 \mathrm{~nm}$ in its WMF between the lipid-free and lipid-bound (DMPC-rHDL) states. Additionally, they observed that the $\alpha$-helicity of bovine apoA-I was 58 or $72 \%$ in the lipid-free and lipid-bound states, respectively. This is consistent with our current results, although we used POPC as a phospholipid in this study. The weakened DMPC clearance activity of bovine and porcine apoA-I positively correlated with the reduced $\alpha$-helicity and the increased molecular number of apoA-I in the POPC-rHDL state, as greater $\alpha$-helicity is necessary to generate larger rHDL particles.

The atheroprotective functions of HDL are characterized by the potent inhibition of LDL oxidation, as well as the expression of endothelial cell adhesion molecules and monocyte chemoattractant protein-1. HDL promotes the efflux of cholesterol from foam cells. In the present study, bovine apoA-I showed the most potent inhibitory activity toward LDL oxidation and macrophage uptake, suggesting that it may be used in nanoparticles for the delivery of therapeutic drugs. The strong inhibition of LDL oxidation by bovine apoA-I (Fig. 4) positively correlated with the decrease in macrophage acLDL phagocytosis (Fig. 5).

Another major function of apoA-I is its antioxidant activity toward LDL. It is well known that the oxidation of LDL participates in the initiation of atherosclerosis and coronary artery disease. Further, the uptake of oxLDL into macrophages, which results in foam cell formation, is an early step in the generation of atherosclerotic plaque. Based on the characterization of rHDL (Table I), bovine apoA-I possesses the greatest capacity to form rHDL among the species, although its minor band size varied. Human apoA-I rHDL contained smaller particles of approximately 80 and $98 \AA$, whereas rHDL containing either bovine or porcine apoA-I exhibited particles of $105 \AA$ (Fig. 2A). Therefore, bovine and porcine apoA-I possibly facilitate the formation of larger rHDL particles with the same molar ratio as greater numbers of apoA-I molecules are required to increase rHDL. Furthermore, bovine apoA-I cross-linking resulted in pentameric structures without any monomeric band, indicating that the majority of bovine apoA-I monomer participated in the cross-linking. These results indicate the enhanced susceptibility of bovine apoA-I to cross-linking. Bovine apoA-I has reportedly been associated with immune function in cattle as reported in the study by Oikawa et al (29). The serum concentration of apoA-I was decreased in cows infected with Salmonella typhimurium. In addition, bovine apoA-I-rHDL had the largest particle size (approximately $105 \AA ̊$ ) (Table I). In our previous study, higher HDL levels were associated with a stronger antioxidant ability compared with lower HDL levels (30). Taken together, these data suggest that the stronger antioxidant ability of bovine apoA-I may be due to the increased particle size. In future studies, structural and functional correlations should be investigated in greater detail.

In conclusion, bovine and porcine apoA-I exhibit unique properties in the lipid-free and lipid-bound (rHDL) states compared with human apoA-I. Specifically, they demonstrated enhanced antioxidant abilities and were resistant to denaturation by urea, suggesting that they may be effective delivery vehicles for therapeutic drugs or vaccines. Future studies are required for the development of encapsulated viral particles in HDL-like structures and to examine their bio-availability in such endeavors. 


\section{Acknowledgements}

This study was supported by the Yeungnam University Research grants in 2010. The authors are grateful for the BK21 plus program of the National Research Foundation for the support of graduate students.

\section{References}

1. Frank PG and Marcel YL: Apolipoprotein A-I: Structurefunction relationships. J Lipid Res 41: 853-872, 2000.

2. Fielding PE and Fielding CJ: Plasma membrane caveolae mediate the efflux of cellular free cholesterol. Biochemistry 34: 14288-14292, 1995.

3. Rye KA and Barter PJ: Antiinflammatory actions of HDL: a new insight. Arterioscler Thromb Vasc Biol 28: 1890-1891, 2008.

4. Kelesidis T, Yang OO, Currier JS, Navab K, Fogelman AM and Navab M: HIV-1 infected patients with suppressed plasma viremia on treatment have pro-inflammatory HDL. Lipids Health Dis 10: 35, 2011.

5. Fryirs MA, Barter PJ, Appavoo M, Tuch BE, Tabet F, Heather AK and Rye KA: Effects of high-density lipoproteins on pancreatic beta-cell insulin secretion. Arterioscler Thromb Vasc Biol 30: 1642-1648, 2010.

6. Park KH, Yun CO, Kwon OJ, Kim CH, Kim JR and Cho KH: Enhanced delivery of adenovirus, using proteoliposomes containing wildtype or V156K apolipoprotein A-I and dimyristoylphosphatidylcholine. Hum Gene Ther 21: 579-587, 2010.

7. Seo J, Yun CO, Kwon OJ, Choi EJ, Song JY, Choi I and Cho KH: A Proteoliposome containing apolipoprotein A-I mutant (V156K) enhances rapid tumor regression activity of human origin oncolytic adenovirus in tumor-bearing zebrafish and mice. Mol Cells 34: 143-148, 2012.

8. Huang R, Silva RA, Jerome WG, Kontush A, Chapman MJ, Curtiss LK, Hodges TJ and Davidson WS: Apolipoprotein A-I structural organization in high-density lipoproteins isolated from human plasma. Nat Struct Mol Biol 18: 416-422, 2011.

9. Bashtovyy D, Jones MK, Anantharamaiah GM and Segrest JP: Sequence conservation of apolipoprotein A-I affords novel insights into HDL structure-function. J Lipid Res 52: 435-450, 2011.

10. Brouillette CG, Anantharamaiah GM, Engler JA and Borhani DW: Structural models of human apolipoprotein A-I: a critical analysis and review. Biochim Biophys Acta 1531: 4-46, 2001.

11. Brewer HB Jr, Ronan R, Meng M and Bishop C: Isolation and characterization of apolipoproteins A-I, A-II, and A-IV. Methods Enzymol 128: 223-246, 1986.

12. Pownall HJ, Massey JB, Kusserow SK and Gotto AM Jr: Kinetics of lipid-protein interactions: interaction of apolipoprotein A-I from human plasma high density lipoproteins with phosphatidylcholines. Biochemistry 17: 1183-1188, 1978.

13. Blois MS: Antioxidant determinations by the use of a stable free radical. Nature 181: 1199-1200, 1958.
14. Cho KH, Park SH, Han JM, Kim HC, Chung YJ, Choi I and Kim JR: A point mutant of apolipoprotein A-I, V156K, exhibited potent anti-oxidant and anti-atherosclerotic activity in hypercholesterolemic C57BL/6 mice. Exp Mol Med 39: 160-169, 2007.

15. Cho KH: Synthesis of reconstituted high density lipoprotein (rHDL) containing apoA-I and apoC-III: the functional role of apoC-III in rHDL. Mol Cells 27: 291-297, 2009.

16. Staros JV: Membrane-impermeant, cleavable cross-linkers: new probes of nearest neighbor relationships at one face of a membrane. Biophys J 37: 21-22, 1982.

17. Chen YH, Yang JT and Martinez HM: Determination of the secondary structures of proteins by circular dichroism and optical rotatory dispersion. Biochemistry 11: 4120-4131, 1972.

18. Park KH, Jang W, Kim KY, Kim JR and Cho KH: Fructated apolipoprotein A-I showed severe structural modification and loss of beneficial functions in lipid-free and lipid-bound state with acceleration of atherosclerosis and senescence. Biochem Biophys Res Commun 392: 295-300, 2010.

19. Markwell MA, Haas SM, Bieber LL and Tolbert NE: A modification of the Lowry procedure to simplify protein determination in membrane and lipoprotein samples. Anal Biochem 87: 206-210, 1978.

20. Esterbauer H, Striegl G, Puhl H and Rotheneder M: Continuous monitoring of in vitro oxidation of human low density lipoprotein. Free Radic Res Commun 6: 67-75, 1989.

21. Noble RP: Electrophoretic separation of plasma lipoproteins in agarose gel. J Lipid Res 9: 693-700, 1968.

22. Fraenkel-Conrat $\mathrm{H}$ : Methods for investigating the essential groups for enzyme activity. Methods Enzymol 4: 247-269, 1957.

23. Cho KH: Biomedicinal implications of high-density lipoprotein: its composition, structure, functions, and clinical applications. BMB Rep 42: 393-400, 2009.

24. Rubin EM, Krauss RM, Spangler EA, Verstuyft JG and Clift SM: Inhibition of early atherogenesis in transgenic mice by human apolipoprotein AI. Nature 353: 265-267, 1991.

25. Newton RS and Krause BR: HDL therapy for the acute treatment of atherosclerosis. Atheroscler Suppl 3: 31-38, 2002.

26. Nicholls SJ, Uno K, Kataoka Y and Nissen SE: ETC-216 for coronary artery disease. Expert Opin Biol Ther 11: 387-394, 2011.

27. Collet X, Marcel YL, Tremblay N, Lazure C, Milne RW, Perret B and Weech PK: Evolution of mammalian apolipoprotein A-I and conservation of antigenicity: correlation with primary and secondary structure. J Lipid Res 38: 634-644, 1997.

28. Jonas A and Krajnovich DJ: Effect of cholesterol on the formation of micellar complexes between bovine A-I apolipoprotein and L-alpha-dimyristoylphosphatidylcholine. J Biol Chem 253: 5758-5763, 1978.

29. Oikawa S, Katoh N, Itoh H, Miyamoto T, Konno M and Kajita T: Decreased serum apolipoprotein A-I concentrations in cows infected with Salmonella typhimurium. Can J Vet Res 61: 182-186, 1997.

30. Lee H, Park JE, Choi I and Cho KH: Enhanced functional and structural properties of high-density lipoproteins from runners and wrestlers compared to throwers and lifters. BMB Rep 42: 605-610, 2009. 